\title{
BMJ Open Modelling the costs and consequences of reducing healthcare-associated infections by improving hand hygiene in an average hospital in England
}

\author{
Julian F Guest (D) , ${ }^{1,2}$ Tomas Keating, ${ }^{1}$ Dinah Gould (D) , ${ }^{3,4}$ Neil Wigglesworth ${ }^{5}$
}

To cite: Guest JF, Keating T, Gould D, et al. Modelling the costs and consequences of reducing healthcare-associated infections by improving hand hygiene in an average hospital in England. BMJ Open 2019;9:e029971. doi:10.1136/ bmjopen-2019-029971

- Prepublication history and additional material for this paper are available online. To view these files, please visit the journal online (http://dx.doi. org/10.1136/bmjopen-2019029971).

Received 20 February 2019

Revised 05 July 2019

Accepted 30 July 2019

\section{Check for updates}

\section{(c) Author(s) (or their} employer(s)) 2019. Re-use permitted under CC BY-NC. No commercial re-use. See rights and permissions. Published by BMJ.

${ }^{1}$ Catalyst Consultants, Rickmansworth, UK

${ }^{2}$ King's College London, London, UK

${ }^{3}$ Cardiff University School of Healthcare Studies, Cardiff, UK ${ }^{4}$ Healthcare Sciences, Cardiff University, Cardiff, South Glamorgan, UK

${ }^{5}$ Directorate of Infection, Guy's \& St Thomas' NHS Foundation Trust, London, UK

\section{Correspondence to} Dr Julian F Guest; julian.guest@catalyst-health. com

\section{ABSTRACT}

Objective To assess the potential clinical and economic impact of introducing an electronic audit and feedback system into current practice to improve hand hygiene compliance in a hypothetical general hospital in England, to reduce the incidence of healthcare-associated infections (HCAls).

Methods Decision analysis estimated the impact of introducing an electronic audit and feedback system into current practice to improve hand hygiene compliance among front-line healthcare practitioners (HCPs). Results The model assumed $4.7 \%$ of adult inpatients (ie, $\geq 18$ years of age) and $1.72 \%$ of front-line HCPs acquire a HCAl in current practice. The model estimated that if use of the electronic audit and feedback system could lead to a reduction in the incidence of HCAls of between $5 \%$ and $25 \%$, then the annual number of HCAls avoided could range between 184 and 921 infections per hospital and HCAl-related mortality could range between 6 and 31 deaths per annum per hospital. Additionally, up to 86 days of absence among front-line HCPs could be avoided and up to 7794 hospital bed days could be released for alternative use. Accordingly, the total annual hospital cost attributable to HCAls could be reduced by between $3 \%$ and $23 \%$, depending on the effectiveness of the electronic audit and feedback system. If introduction of the electronic audit and feedback system into current practice could lead to a reduction in the incidence of HCAls by at least $15 \%$, it would have $a \geq 0.75$ probability of affording the National Health Service (NHS) a cost-effective intervention.

Conclusion If the introduction of the electronic audit and feedback system into current practice in a hypothetical general hospital in England can improve hand hygiene compliance among front-line HCPs leading to a reduction in the incidence of HCAls by $\geq 15 \%$, it would potentially afford the NHS a cost-effective intervention.

\section{INTRODUCTION}

Healthcare-associated infections (HCAIs) are those occurring in a healthcare setting that were not present prior to a patient entering that care setting. ${ }^{1}$ HCAIs can develop either as a direct result of healthcare interventions or from being in contact with a healthcare setting. ${ }^{2}$ The epidemiological evidence indicates that hand-mediated transmission is a

\section{Strengths and limitations of this study}

- This is the first published study to evaluate the potential clinical and economic impact of reducing the incidence of healthcare-associated infections (HCAls) in a hypothetical general hospital in England.

- This was a modelling study based on disparate sources of published evidence.

- The costs and consequences of introducing an electronic audit and feedback system into current practice to improve hand hygiene compliance among front-line healthcare professionals (HCPs) in an average general hospital in England were estimated from a model depicting the pathways and associated management of adult inpatients and front-line HCPs who acquire a HCAl in the hospital environment over a period of 1 year.

- The relationship between HCPs improving compliance with hand hygiene and reducing the background incidence of HCAls is uncertain.

- The model does not consider HCPs' adaptation to the electronic audit and feedback system and subsequent decline in compliance nor does it distinguish between different types of pathogens causing HCAls and the definition of HCAl would appear to vary between clinicians.

major contributing factor in the acquisition and spread of infection in hospitals, and such transmission can occur directly via hands, or indirectly via an environmental source (eg, commodes, wash bowls, blood pressure monitors, mobile phones).${ }^{34}$ HCAIs can be caused by a range of pathogens including meticillin-resistant Staphylococcus aureus (MRSA), meticillin-sensitive Staphylococcus aureus, Clostridium difficile ( $C$. difficile) and Escherichia $\operatorname{coli}(\text { E. coli) })^{5}$ Antibiotics are the mainstay of HCAI management ${ }^{6}$ although their use can increase the likelihood of infection from drug-resistant organisms and C. difficile. ${ }^{67}$

HCAIs pose a serious risk to patients, healthcare practitioners (HCPs) and visitors in a healthcare setting. Within a general 


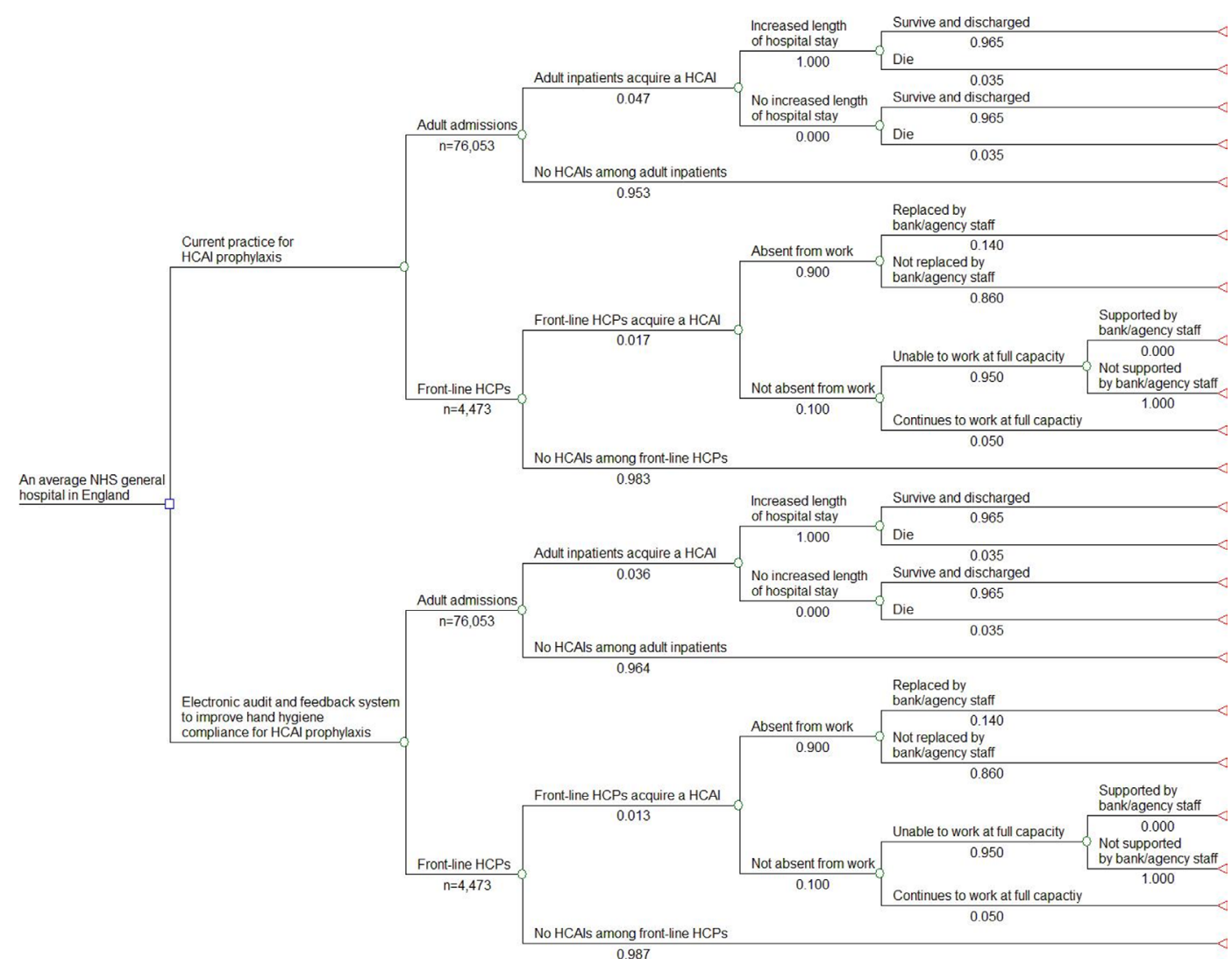

Figure 1 Decision model depicting the management of HCAls in an average NHS general hospital. The number below each branch depicts the probability of following a particular pathway, and is based on a $25 \%$ reduction in the incidence of HCAls following the introduction of an electronic audit and feedback system into current practice to improve hand hygiene compliance among front-line HCPs. HCAl, healthcare-associated infection; HCP, healthcare practitioner; NHS, National Health Service.

hospital, HCAIs are more likely to occur among patients who become vulnerable to infection due to several factors, such as extended and inappropriate use of invasive devices and antibiotics, undergoing a high-risk and sophisticated procedure, being immunocompromised and other severe underlying conditions. ${ }^{8}$ Inadequate infection control expertise and insufficient hygiene levels can also be contributing factors. ${ }^{8}$

Public Health England monitors the epidemiology of certain HCAIs through routine surveillance programmes, and also advises on how to prevent and control infection in establishments, such as hospitals. ${ }^{9}$ All National Health Service (NHS) hospitals must have an infection prevention service in place. Evidence-based guidelines for preventing HCAIs in NHS hospitals in England state that HCPs need to apply standard infection control precautions to the care of all patients. ${ }^{10}$ The guidelines also affirm that one aspect of infection control is the consistent adherence to a hygiene protocol comprising: hospital environmental hygiene; hand hygiene; use of personal protective equipment; safe use and disposal of sharps; and principles of asepsis. ${ }^{10}$

These guidelines specify that a hand hygiene protocol requires HCPs to decontaminate their hands: (1) immediately before each episode of direct patient contact or care, including clean/aseptic procedures; (2) immediately after each episode of direct patient contact or care;
(3) immediately after contact with body fluids, mucous membranes and non-intact skin; (4) immediately after other activities or contact with objects and equipment in the immediate patient environment that may result in the hands becoming contaminated; and (5) immediately after the removal of gloves. ${ }^{10}$ This hand hygiene protocol is consistent with the World Health Organization's (WHO) 'My 5 Moments of Hand Hygiene'. ${ }^{11}$

Audit with performance feedback is an important component of the WHO's hand hygiene guidelines ${ }^{4}$ and is undertaken routinely in many high and low income countries. Audit with feedback can improve the uptake of healthcare interventions generally ${ }^{12}$ and is an important component of many hand hygiene interventions. ${ }^{13}$ High levels of hand hygiene adherence are frequently overestimated $^{14}$ as a result of the Hawthorne effect ${ }^{15}$ and other sources of bias. ${ }^{14}$ Awareness of being watched encourages HCPs to cleanse their hands more often ${ }^{14} 1617$ while audit periods are typically brief (15-20 min) giving an incomplete picture of usual practice, especially if intricate procedures with high risk of hand contamination are postponed until the audit is over. ${ }^{17}$

There have been numerous systematic reviews assessing the risks and impact of HCAIs (too many to quote) in both high and low income countries. In particular, two systematic reviews explored the effectiveness of automated hand hygiene monitoring systems, and both 
included electronic devices. Both reviews concluded that such systems may be of benefit, but acknowledged that the primary studies were poorly controlled (pre/post test designs) and both recommended an economic analysis. ${ }^{1819}$ There is minimal published evidence on the association between HCAIs and hand hygiene, ${ }^{13}$ so it is hard to quantify the effect of improving hand hygiene on the rate of acquiring HCAIs. However, one study in Geneva between 1994 and 1998 saw hand hygiene compliance increase from $48 \%$ to $66 \%$, resulting in a reduction in the annual HCAI prevalence, from $16.9 \%$ in 1994 to $9.9 \%$ in $1998 .^{20}$ Against this background, this study considers the potential cost-effectiveness of reducing the incidence of HCAIs by varying amounts following the introduction of a 'hand hygiene electronic audit and feedback system' into current practice to improve hand hygiene compliance among front-line HCPs in a hypothetical general hospital in England.

\section{METHODS}

\section{Study design}

This was a modelling study which estimated the potential clinical and economic impact of reducing the incidence of HCAIs in a hypothetical general hospital in England, by improving compliance with hand hygiene protocols among front-line HCPs.

\section{Patient and public involvement}

Patients and members of the public were not directly involved in this study.

\section{Data sources}

A systematic literature review was performed by searching PubMed, the Kings Fund, House of Commons library, NHS digital (Hospital Episode Statistics, workforce statistics and bed occupancy data) and the Office for National Statistics for relevant publications on HCAIs published between January 2008 and June 2018. The search focused on epidemiology, clinical and health outcomes, management, resource use, costs and productivity and the search strings used to identify publications contained terms such as: 'Hospital acquired infection' OR 'Nosocomial infection' OR 'Healthcare associated infection' OR 'HAI' OR 'HCAI' AND 'epidemiology' OR 'prevalence' OR 'incidence' OR 'mortality' OR 'Cost' OR 'Cost-effectiveness'. The search was limited to publications in the English language and only studies concerning humans. If multiple sets of the same data were found over the 10 year period then the most recent published data set was used. Manual searches were also performed based on citations in the published articles and suggestions from the clinical co-authors.

The searches yielded 1104 different publications, of which 905 were excluded because of duplication or lack of relevance generating 199 publications to review in full. Of the 199 publications which were reviewed, a further 100 were excluded as they did not meet the study criteria. This left 99 articles for data extraction of which 44 contained relevant data which were used to inform this modelling study.

\section{Health economic modelling}

A decision model was constructed depicting the pathways and associated management of adult inpatients and front-line HCPs who acquire a HCAI in the hospital environment over a period of 1 year (figure 1). The model considers the costs and consequences of current practice compared with introducing an electronic audit and feedback system to improve hand hygiene compliance among front-line HCPs in an average general hospital in England. The model considers the impact of these two strategies on HCAI prophylaxis among both adult inpatients and front-line HCPs.

\section{Healthcare resource use}

The model incorporated the following assumptions (table 1):

- In an average year in an average NHS hospital in England, there would be 76053 adult admissions, ${ }^{21}$ 510 adult inpatient beds ${ }^{22}$ and 4473 front-line HCPs. ${ }^{23}$

- $4.7 \%$ of adult inpatients would acquire a HCAI. ${ }^{24}$

- $3.5 \%$ of patients who acquire a HCAI would die from their infection. ${ }^{25}$

- $1.72 \%$ of front-line HCPs would acquire a HCAI (an estimated mean from two different sources). ${ }^{26} 27$

- Every adult inpatient who acquires a HCAI would have an increased length of stay.

- Once an adult inpatient acquires a HCAI, $90 \%$ would remain on the ward to which they were admitted, $10 \%$ would be isolated ${ }^{28}$ and $0.04 \%$ would be transferred into intensive care (ICU). ${ }^{21} 2930$

- Patients would remain on the ward or in an isolation unit for a mean of 9.1 days. $^{29}$

- Patients who are transferred to ICU would remain there for a mean of 4.0 days before returning to the ward. ${ }^{30}$

Healthcare resource use and corresponding costs pertaining to bed occupancy and outpatient visits are detailed in tables 1 and 2. The daily cost of bed occupancy at 2016/2017 prices (table 2) was applied to the additional length of hospital stay to estimate the cost of hospital stay attributable to HCAIs. The costs of diagnostic tests, prescribed medication and clinician time were assumed to be included in the daily cost of hospital stay.

\section{HCPs absenteeism from work}

According to the clinical authors, it is NHS Hospital Trust policy that HCPs who develop an infection should not attend work. The model assumed that $90 \%$ of front-line HCPs who acquire a HCAI would not attend work for a mean of 5 days, while the other $10 \%$ would attend.

The cost of front-line HCPs being absent from work due to HCAIs comprises the cost of replacing HCPs who acquire a HCAI with either bank or agency staff (table 2). The cost of replacing front-line HCPs with bank staff was calculated using a weighted average of the cost per day per staff type weighted by the proportion of the different types/grades of front-line staff that work in NHS hospitals in England. ${ }^{23}$ The cost of agency staff was assumed to be 
Table 1 Model inputs

\begin{tabular}{|c|c|c|}
\hline Parameter & Value & Source \\
\hline \multicolumn{3}{|l|}{ Probabilities } \\
\hline Probability of patients acquiring a $\mathrm{HCAl}$ & 0.047 & 24 \\
\hline Probability of patients dying as a result of a HCAI & 0.035 & 25 \\
\hline Probability of $\mathrm{HCPs}$ acquiring a $\mathrm{HCAl}$ & 0.017 & 2627 \\
\hline Probability of HCPs being absent from work due to acquiring a HCAI & 0.900 & (Assumption) \\
\hline Probability of absent HCPs being replaced by agency or bank staff & 0.140 & 53 \\
\hline Probability of HCPs not being absent from work but unable to work at full capacity & 0.950 & (Assumption) \\
\hline $\begin{array}{l}\text { Probability of HCPs not being absent from work and being supported by bank or } \\
\text { agency staff }\end{array}$ & 0.000 & (Assumption) \\
\hline $\begin{array}{l}\text { Probability of adult inpatients having increased length of hospital stay as a result } \\
\text { of a HCAl }\end{array}$ & 1.000 & (Assumption) \\
\hline Probability of HCPs being admitted into hospital as a result of acquiring a $\mathrm{HCAl}$ & 0.001 & (Assumption) \\
\hline Probability of being placed in isolation following a HCAI & 0.100 & 28 \\
\hline Probability of being transferred to an ICU as a result of a $\mathrm{HCAI}$ & 0.0004 & 212930 \\
\hline Probability of remaining on a ward after acquiring a HCAI & 0.900 & (Calculation) \\
\hline Probability of a follow-up hospital outpatient appointment & 0.310 & 295455 \\
\hline \multicolumn{3}{|l|}{ Resource use } \\
\hline Probable additional number of bed days as a result of a HCAI & 9.100 & 29 \\
\hline Number of follow-up hospital outpatient appointments & 0.800 & 56 \\
\hline Average number of days spent in critical care & 3.970 & 30 \\
\hline Length of time HCPs are off work and replaced by agency staff (days) & 5.000 & (Assumption) \\
\hline
\end{tabular}

$\mathrm{HCAl}$, healthcare-associated infection; HCP, healthcare practitioner; ICU, intensive care unit.

the maximum $55 \%$ above the daily cost of NHS employees, excluding any administrative fees. ${ }^{31}$ The model assumed that $14 \%$ of absent HCPs would be replaced by agency or bank staff, of which $70 \%$ would be bank staff and the other $30 \%$ would be agency staff.

\section{Hand hygiene electronic audit and feedback system}

The model assumed the electronic audit and feedback system comprises a technology (such as 'Tork Vision Hand Hygiene') which monitors compliance with hand hygiene practices according to the WHO's five moments for hand hygiene (ie, moments 1,4 and 5). ${ }^{11}$ This is accomplished by tracking HCPs' movements with high

\begin{tabular}{lrl}
\hline Table 2 & Hospital resource costs at 2016/2017 & prices \\
\hline Resource & Cost (£) & Source \\
\hline $\begin{array}{l}\text { General ward cost per bed day } \\
\text { General ward cost per excess bed } \\
\text { day }\end{array}$ & 586.59 & 57 \\
\hline Isolation ward cost per day & 586.00 & 57 \\
ICU cost per day & 1621.16 & 57 \\
\hline Hospital outpatient cost per visit & 201.00 & 57 \\
NHS bank staff per day & 286.19 & 58 \\
\hline Agency staff per day & 443.59 & 31 \\
\hline
\end{tabular}

ICU, intensive care unit; NHS, National Health Service. precision in real time and by combining their use of hand sanitisers (USAGE events) with their physical movements in relation to patients' beds (OPPORTUNITY events). The compliance results are fed back to the HCPs both at a group and individual level. The system incorporates the use of antennae mounted in the ceilings in hospital wards, tags worn by HCPs and hand hygiene product dispenser tags that record dispensing actions.

One feasibility study showed that the electronic audit and feedback system led to a $20 \%$ increase in compliance with hand hygiene practices (ie, cleaning hands before patient contact, cleaning hands after patient contact and cleaning hands after contact with patient surroundings (WHO's Hand Hygiene moments 1, 4 and 5). ${ }^{32}$ The base case model assumes that introducing the electronic audit and feedback system into current practice to improve compliance with hand hygiene protocols would lead to a reduction in the incidence of HCAIs by $25 \%$. However, the effect of reducing the incidence of HCAIs by $20 \%, 15 \%, 10 \%$ and $5 \%$ has also been evaluated. The model assumes that the electronic audit and feedback system would cost an average hospital in England £1.50 per inpatient bed per day.

\section{Model outputs}

The model outputs for an average NHS hospital with 76053 adult admissions, ${ }^{21} 510$ adult inpatient beds ${ }^{22}$ and 4473 front-line $\mathrm{HCPs}^{23}$ were:

- The annual number of HCAIs avoided. 
- The annual number of HCAI-related patient deaths avoided.

- The annual number of HCAI-related days of avoided absence by HCPs.

- The annual number of HCAI-related occupied bed days.

- The total annual hospital cost attributable to HCAIs comprising:

- The annual cost of patient management.

- The annual cost of HCP management.

- The annual cost of agency staff.

- The annual cost of the electronic audit and feedback system to improve hand hygiene compliance.

\section{Economic evaluation}

The cost-effectiveness of introducing the electronic audit and feedback system into current practice was calculated by dividing the cost difference between the two hand hygiene strategies by the difference in health outcomes of the two strategies. If use of the electronic audit and feedback system leads to an improvement in outcome for less cost, it was considered a dominant (cost-effective) strategy.

The cost-benefit (return on investment) of introducing the electronic audit and feedback system into current practice was also calculated by dividing the net financial benefit of the strategy by the cost of implementing the electronic audit and feedback system.

\section{Sensitivity analysis}

Probabilistic sensitivity analysis was undertaken to evaluate uncertainty within the model by applying a distribution to all the model inputs. A beta distribution was assigned to the probabilities and a gamma distribution to resource use and costs, by assuming a $10 \% \mathrm{SE}$ around the mean values. This enabled the generation of 10000 iterations of the model by randomly selecting a value from all the different inputs simultaneously. The outputs from these iterations was a distribution of costs and outcomes over 1 year for each strategy. The analysis also estimated the probability of implementing the electronic audit and feedback system to improve hand hygiene compliance among front-line HCPs being cost-effective when compared with current practice.

Deterministic sensitivity analysis was also performed to examine the effect of individually decreasing and increasing by $25 \%$ the value of each of the key model inputs and assumptions.

\section{RESULTS}

\section{HCAI-related outcomes}

The base case model estimated that in an average NHS hospital with 76053 adult admissions per annum, ${ }^{21} 510$ adult inpatient beds ${ }^{22}$ and 4473 front-line $\mathrm{HCPs}^{23}$ there are 3683 HCAIs per annum. Introduction of the electronic audit and feedback system into current practice was estimated to potentially reduce this to 2762 HCAIs per annum (table 3). The model also estimated that there are 126 HCAI-related patient deaths per annum in an average NHS hospital and this could potentially be reduced to 95 deaths following introduction of the electronic audit and feedback system into current practice. Hence, introduction of the electronic audit and feedback system into current practice could potentially lead to the avoidance of 921 HCAIs and 31 HCAI-related patient deaths and free-up 7794 hospital bed days for alternative use, equivalent to $4 \%$ of the annual number of hospital bed days. Additionally, 86 days of front-line HCPs being absent from work could be avoided (table 3). These estimates can vary according to the percentage reduction in HCAIs following introduction of the electronic audit and feedback system into current practice (table 3).

\section{HCAI-related hospital costs}

The base case model estimated that an average NHS general hospital with 76053 adult admissions per annum, ${ }^{21} 510$ adult inpatient beds ${ }^{22}$ and 4473 front-line $\mathrm{HCPs}^{23}$ incurs an annual cost of $£ 11.9$ million attributable to HCAIs, of which $>99 \%$ is attributable to patient management (table 4 ). This could potentially be reduced by $23 \%$ ( $£ 2.7$ million) following introduction of the electronic audit and feedback system into current practice (table 4). These estimates can vary according to the percentage reduction in HCAIs following introduction of the electronic audit and feedback system into current practice (table 5).

\section{Cost-effectiveness analysis}

Outputs from the model showed that introduction of the electronic audit and feedback system into current practice is expected to lead to a cost decrease and a corresponding increase in outcomes for an average hospital with 76053 adult admissions per annum, ${ }^{21} 510$ adult inpatient beds ${ }^{22}$ and 4473 front-line HCPs. ${ }^{23}$ Thus, use of the electronic audit and feedback system potentially affords the NHS a cost-effective intervention, since it could lead to an improvement in outcomes for less cost.

\section{Cost-benefit analysis}

The net benefit of introducing the electronic audit and feedback system into current practice varied between $£ 33800$ and £2.4 million, depending on the percentage reduction in the incidence of HCAIs (table 6). The analyses showed that if the percentage reduction in the incidence of HCAIs is $\geq 3 \%$, then the cost of introducing the electronic audit and feedback system into current practice in an average general hospital would be offset by the ensuing cost-reductions. The cost-benefit ratio showed that for every $£ 1$ spent by an average general hospital on the electronic audit and feedback system, it could benefit from a reduction in costs of $\geq £ 1.10$ attributable to HCAIs (table 6).

\section{Sensitivity analyses}

Probabilistic sensitivity analysis highlighted the distribution in the incremental costs and outcomes at 12 months between the two hand hygiene strategies (figure 2). 


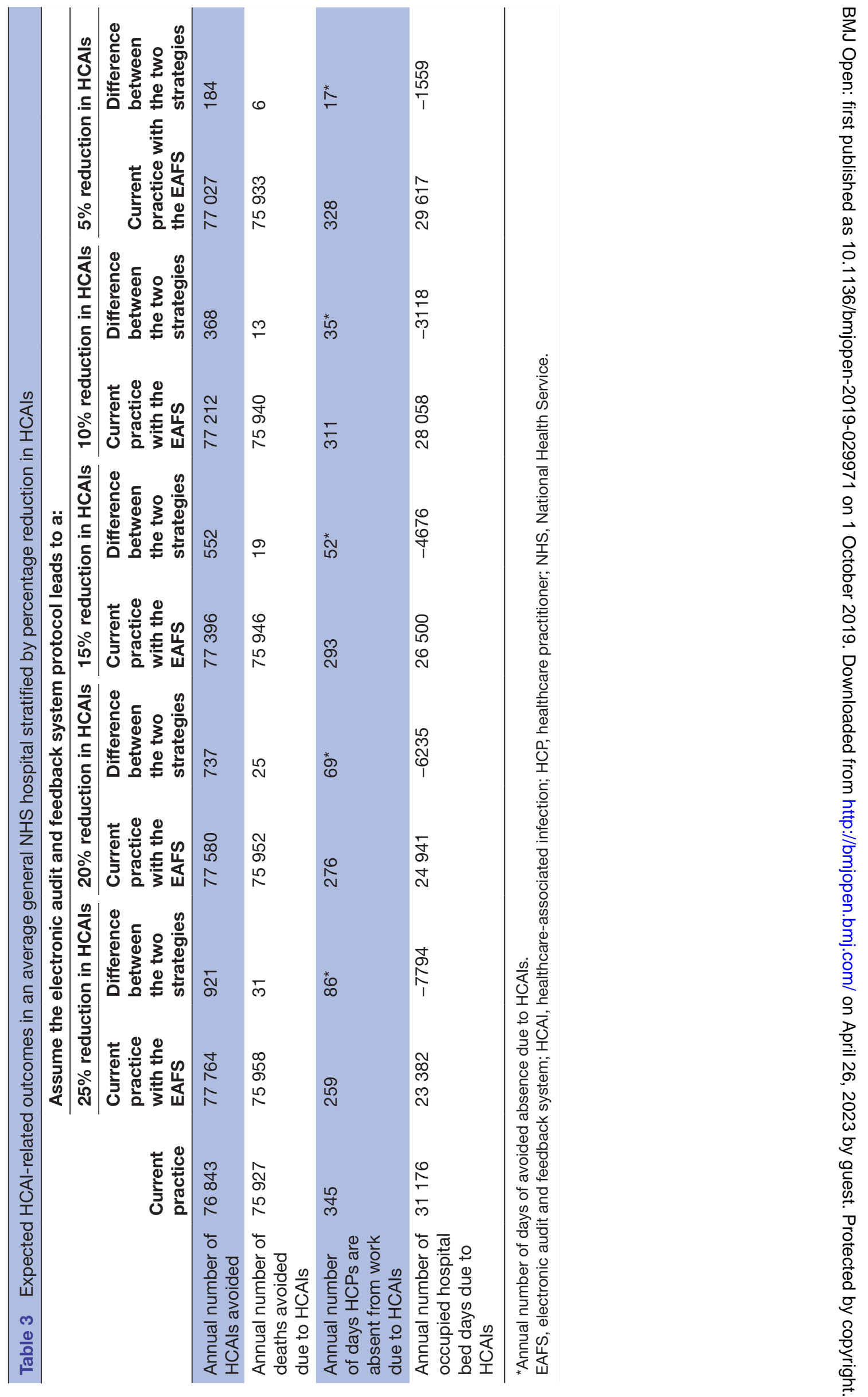


Table 4 Expected costs in an average general NHS hospital attributable to HCAls (assumes the electronic audit and feedback system protocol leads to a $25 \%$ reduction in HCAls)

In an average NHS hospital with 76053 adult admissions per annum,

4473 HCPs and 510 adult inpatient beds

\begin{tabular}{|c|c|c|c|}
\hline & Current practice & $\begin{array}{l}\text { Current practice with } \\
\text { the electronic audit and } \\
\text { feedback system }\end{array}$ & $\begin{array}{l}\text { Cost difference between the } \\
\text { two strategies }\end{array}$ \\
\hline $\begin{array}{l}\text { Annual cost of patient management } \\
\text { for HCAls }\end{array}$ & $£ 11858527$ (99) & £8 893895 (97) & $-£ 2964632$ \\
\hline $\begin{array}{l}\text { Annual cost of HCP management for } \\
\text { HCAls }\end{array}$ & $£ 369(<1)$ & $£ 277(<1)$ & $-£ 92$ \\
\hline $\begin{array}{l}\text { Annual cost of the electronic audit } \\
\text { and feedback system }\end{array}$ & & $£ 278958$ (3) & $£ 278958$ \\
\hline $\begin{array}{l}\text { Total annual hospital cost } \\
\text { attributable to HCAls }\end{array}$ & $£ 11878053(100)$ & $£ 9189209$ (100) & $-£ 2688844$ \\
\hline
\end{tabular}

Per cent of total cost in parentheses.

$\mathrm{HCAl}$, healthcare-associated infection; HCP, healthcare practitioner; NHS, National Health Service.

Outputs from the analysis suggest that use of the electronic audit and feedback system has to lead to a reduction in the incidence of HCAIs by at least $15 \%$ in order to afford the NHS an intervention which has a sufficiently high probability of being cost-effective (ie, $\geq 0.75$ ) (figure 3). In the base case model, the probability of the electronic audit and feedback system being cost-effective was $\geq 0.90$ (figure 3 ).

Deterministic sensitivity analysis (table 7) was performed on the base case model (ie, by assuming the electronic audit and feedback system leads to a $25 \%$ reduction in the incidence of HCAIs). The analysis suggests that the probability of the electronic audit and feedback system affording the NHS a cost-effective intervention is primarily sensitive to:

- Its ability to reduce the incidence of HCAIs.

- Probability of patients acquiring a HCAI.

- Annual number of adult admissions into an average hospital.
- Average additional length of hospital stay after acquiring a HCAI.

The probability of the electronic audit and feedback system affording the NHS a cost-effective intervention was found to be less sensitive to changes in the other model inputs. In particular, increasing the cost of the electronic audit and feedback system has negligible effect on the results since it accounts for only $3 \%$ of the total cost attributable to HCAIs incurred by an average NHS general hospital in the base case model.

\section{DISCUSSION}

This study estimated that in an average NHS hospital with 76053 adult admissions per annum, ${ }^{12} 510$ adult inpatient beds ${ }^{13}$ and 4473 front-line HCPs, ${ }^{14}$ there are 3683 HCAIs per annum, and as a consequence 31176 bed days are occupied by patients with these infections. This equates to $17 \%$ of the annual number of all bed days in an average

Table 5 Expected costs in an average general NHS hospital attributable to HCAls stratified by percentage reduction in HCAls

Total annual hospital cost attributable to HCAls in an average NHS hospital with 76 053 adult admissions per annum, 4473 HCPs and 510 adult inpatient beds

\begin{tabular}{llll}
$\begin{array}{l}\text { Percentage reduction in HCAls } \\
\text { attributable to the electronic } \\
\text { audit and feedback system }\end{array}$ & Current practice & $\begin{array}{l}\text { Current practice with } \\
\text { the electronic audit and } \\
\text { feedback system }\end{array}$ & $\begin{array}{l}\text { Cost difference between the } \\
\text { two strategies }\end{array}$ \\
\hline $25 \%$ & $£ 11878053$ & $£ 9189209$ & $-£ 2688844$ \\
\hline $20 \%$ & $£ 11878053$ & $£ 9783226$ & $-£ 2094827$ \\
$15 \%$ & $£ 11878053$ & $£ 10377243$ & $-£ 1500810$ \\
\hline $10 \%$ & $£ 11878053$ & $£ 10971259$ & $-£ 906794$ \\
\hline $5 \%$ & $£ 11878053$ & $£ 11565276$ & $-£ 312777$ \\
\hline
\end{tabular}

HCAI, healthcare-associated infection; HCP, healthcare practitioner; NHS, National Health Service. 


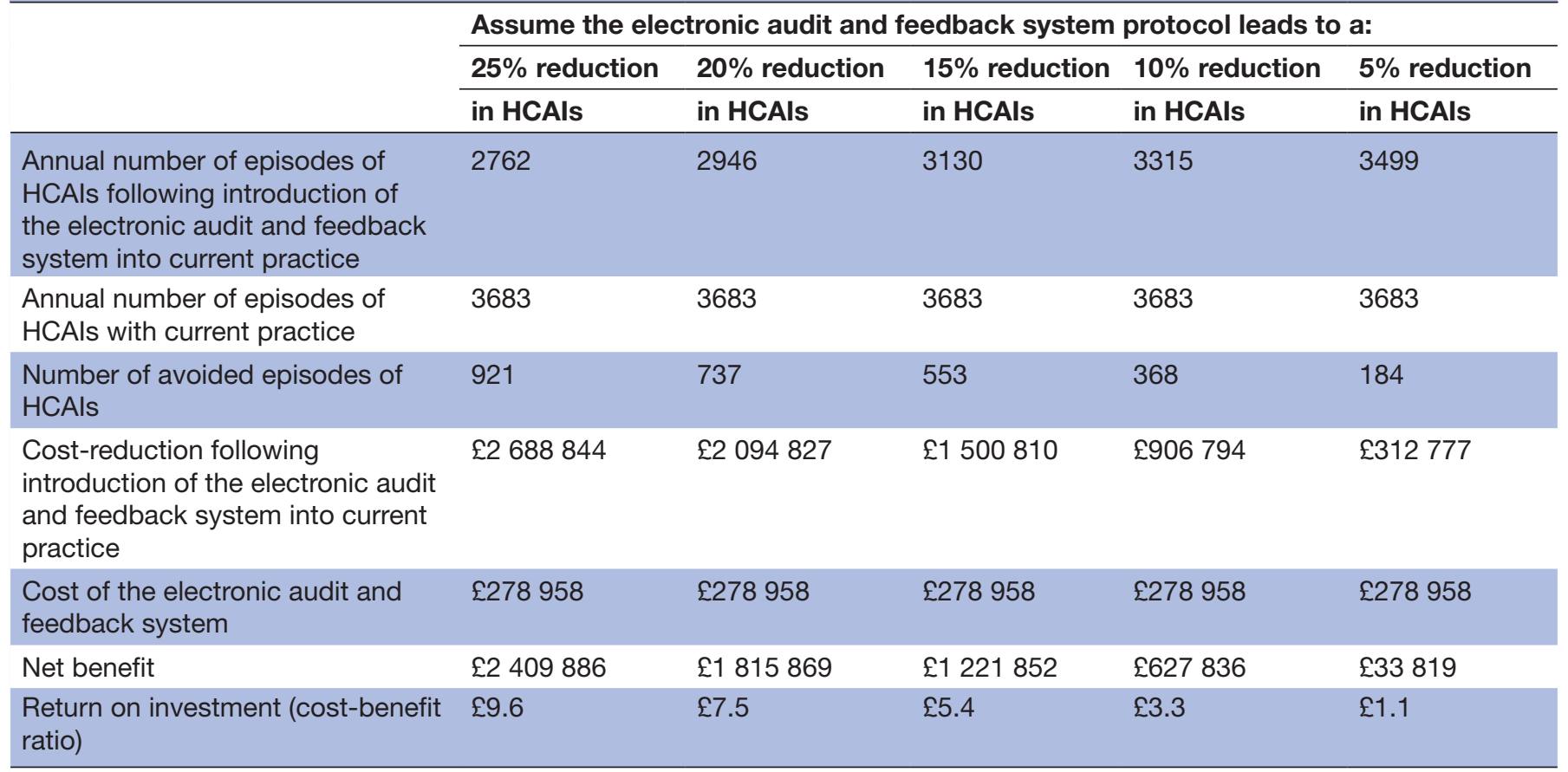

HCAl, healthcare-associated infection.

NHS hospital. Furthermore, if the mean additional length of hospital stay as a result of a HCAI was reduced by half (from 9.1 days $^{19}$ to 4.5 days), $8 \%$ of all bed days would still be occupied by patients with a HCAI. There is an opportunity cost to this HCAI-related bed occupancy. There has been an increase in hospital bed occupancy in England in recent years, due in part to the increased levels of hospital activity. ${ }^{33}$ Consequently, this impacts on the speed with which patients can be admitted into hospital. It has also been shown to be a significant factor in the deterioration in performance against the 4-hour standard for admitting or discharging patients from hospital accident and emergency departments. ${ }^{33}$ The shortage of available beds has also contributed to patients being admitted to hospitals outside their local area, which may delay their recovery. ${ }^{33}$ Hence, this study may have underestimated the potential cost-effectiveness of the electronic audit and feedback system, when one considers its potential to result in a decrease in the number of HCAI-related occupied bed days and thereby facilitate an increase in the speed of hospital admissions.

WHO guidance highlights tuberculosis as an occupational risk factor for $\mathrm{HCPs}^{34}$; they are also at risk of acquiring bloodborne infections (eg, HIV, hepatitis B and C). While HCPs may not be at high-risk of 'classic' HCAIs, they are at risk of community-acquired infections brought into a hospital by patients. These include gastrointestinal infections (eg, norovirus) and respiratory infections (eg, influenza, respiratory syncitial virus). Rarely, HCPs may also develop varicella, measles or mumps through patient contact. Risks of acquiring a HCAI are higher for HCPs than for members of the public visiting a hospital because they have more patient contacts than visitors, which are sustained over a longer period of time. Poor hand hygiene plays an important role in the spread of gastrointestinal infections. ${ }^{35-37}$ Accordingly, the model estimated that $1.72 \%$ of front-line HCPs would acquire a HCAI. ${ }^{26} 27$ Sensitivity analyses showed that changing this value has negligible impact on the study's findings.

This study is predicated on an electronic audit and feedback system which monitors compliance with hand hygiene practices according to the WHO's Moments 1, 4 and $5^{10}$ which are designed to reduce transmission of pathogens between patients. This study showed that introducing such an electronic audit and feedback system into current practice to improve hand hygiene compliance in a hypothetical general hospital in England is potentially cost-effective providing it leads to a $\geq 15 \%$ reduction in the incidence of HCAIs. There is substantial evidence that increasing hand hygiene compliance is associated with reducing HCAIs, although uncertainty remains about the proportion of HCAIs that can be prevented by improving hand hygiene compliance. ${ }^{4}$ Moreover, it has been suggested that estimating hand hygiene compliance with the WHO's Moments 1, 4 and 5 by monitoring leads to reasonable estimates of overall compliance with all 5 Moments. ${ }^{38}{ }^{39}$ Limitations with the published evidence are due to the fact that designing and conducting robust, ethical, randomised controlled trials in the field of hand hygiene is challenging. ${ }^{13}$ Consequently, the recommendations on hand hygiene protocols are based on evidence from non-randomised controlled studies, quasi-experimental studies, observational studies and laboratory studies with volunteers. ${ }^{10}$ 


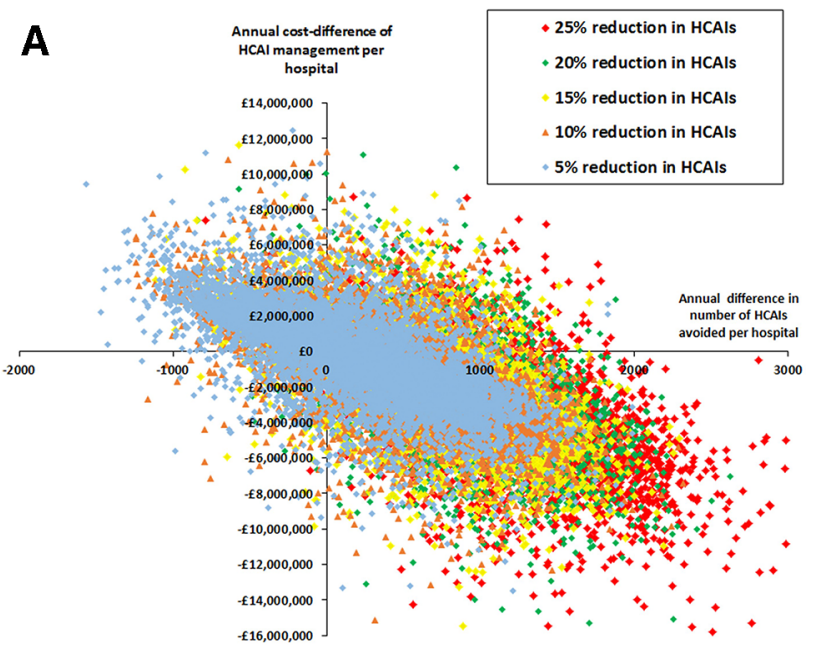

C

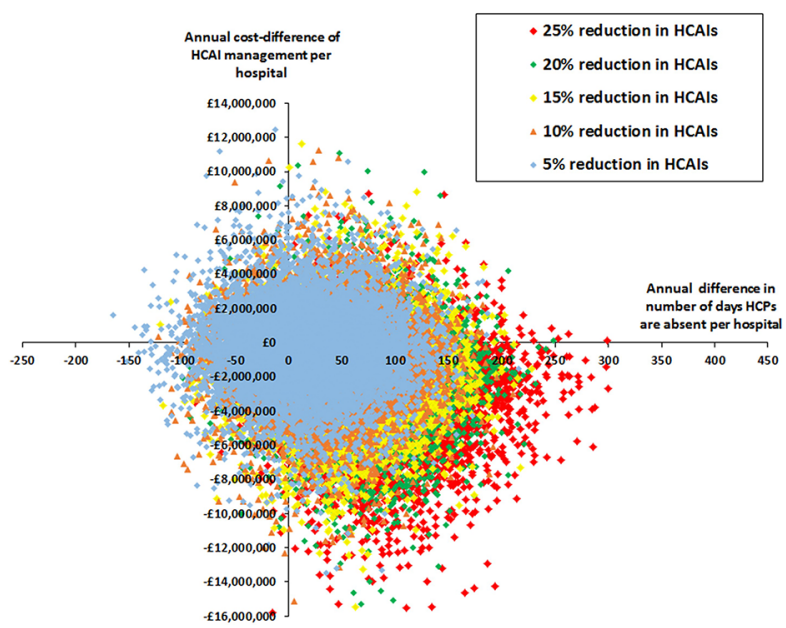

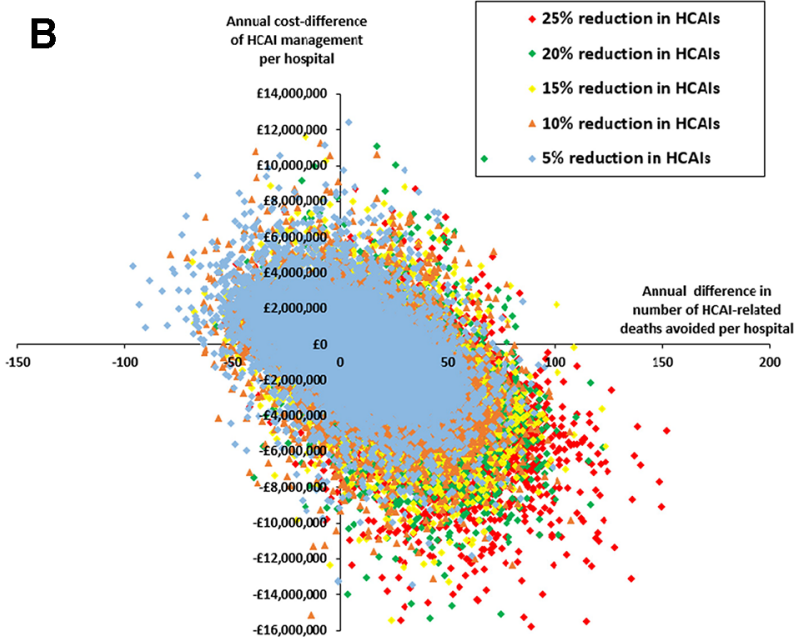

D

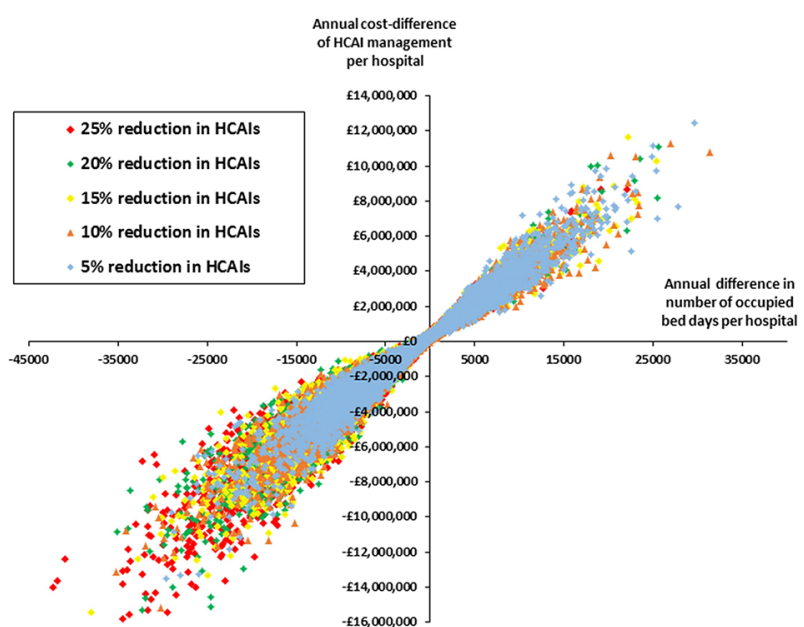

$-\$ 16,000,000$

Figure 2 Scatterplot of the incremental costs and outcomes between the two strategies in 1 year in an average general hospital following 10000 iterations of the model, stratified by reduction in HCAl incidence associated with the electronic audit and feedback system. (A) HCAls avoided. (B) Patient deaths avoided. (C) Days HCPs are absent from work. (D) Occupied hospital bed days. HCAl, healthcare-associated infection; HCP, healthcare practitioner.

There have been few studies assessing the health economic impact of hand hygiene protocols within healthcare settings. One review of economic studies on the impact of alcohol-based hand hygiene products in healthcare organisations ${ }^{40}$ concluded that, while further research is required to measure the direct impact of improved hand hygiene on infection rates, the potential benefit of providing alcohol-based handrubs is likely to outweigh costs, and their wide-scale promotion should continue. Many of the older studies are not directly comparable to our study since they have examined direct costs of alternative handrubs, whereas this study has assessed the costs of introducing an electronic audit and feedback system into current practice to improve hand hygiene compliance with the clinical consequences and associated costs as they pertain to both patients (eg, increased length of hospital stay) and HCPs (eg, absenteeism from work).
One recent study has examined the cost-effectiveness of multi-modal hospital interventions to improve hand hygiene compliance in a middle-income country. ${ }^{41}$ However, this study was restricted to assessing the relationship between hand hygiene compliance and preventing a MRSA blood stream infection in ICU settings. Other economic studies in high-income countries ${ }^{20} 4243$ reported the potential for a hand hygiene intervention to be cost-effective if the incidence of HCAIs was reduced by at least $1 \%$, without reporting the probability of the intervention being cost-effective. Our study also suggests that introducing an electronic audit and feedback system into current practice to improve hand hygiene compliance would be cost-saving and cost-effective if the incidence of HCAIs was reduced by at least $3 \%$. However, the probability of it being cost-effective is $\geq 0.75$ if it leads to $\mathrm{a} \geq 15 \%$ reduction in the incidence of HCAIs. Nevertheless, it is difficult to compare these studies with our own 
Probability of the

electronic audit and

feedback system being cost-effective

1.00

0.80

0.60

0.40

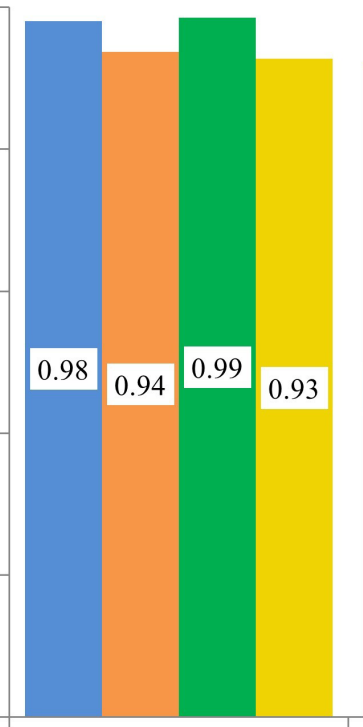

$25 \%$ reduction in HCAIs

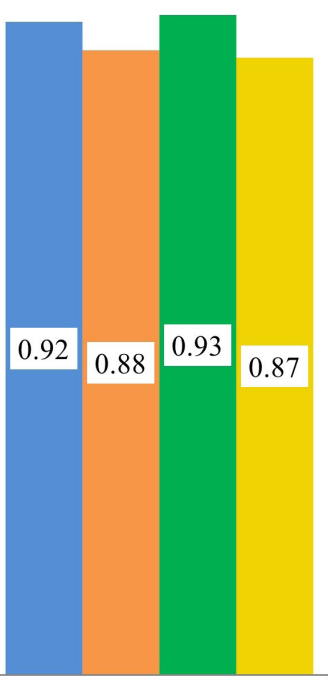

$20 \%$ reduction in HCAIs
- HCAIs avoided

- Patient deaths avoided

- Avoided absenteeism among HCPs

$\square$ Occupied bed days avoided

Figure 3 Probability of the electronic audit and feedback system being cost-effective compared with current practice, stratified by reduction in $\mathrm{HCAl}$ incidence. $\mathrm{HCAl}$, healthcare-associated infection; HCP, healthcare practitioner.

due to different study designs and other methodological differences.

Observational studies of hand hygiene behaviour indicate an abundant risk of exogenous (cross) infection as HCPs move rapidly between patients, often without undertaking adequate hand hygiene. ${ }^{44} 45$ Additionally, the ICU environment is heavily contaminated with hospital strains of pathogenic organisms which have been shown to cause infection. ${ }^{46}$ Increased hand hygiene performance has been shown to reduce endogenous infection. ${ }^{47}$ Improving HCPs' behaviour in infection prevention and control is challenging, since their behaviour is influenced, in part, by individual preferences and a degree of professional isolation. ${ }^{49}$ Clearly, for any hand hygiene protocol to be clinically effective and cost-effective, HCPs' adherence to hand hygiene guidelines should be regularly audited, and the findings should be reported back in order to improve and sustain high levels of compliance. Additionally, healthcare organisations should provide regular training in risk assessment. A recent review concluded that the effectiveness of auditing and feedback depends on HCPs' baseline performance and how feedback is provided to them. ${ }^{50}$ Moreover, such electronic audit and feedback can lead to small, but potentially important improvements in HCPs' practice. ${ }^{50}$ Patients, relatives and other visitors should also be provided with information about the importance of hand hygiene and how to keep their own hands clean.

\section{Study limitations}

This health economic assessment of introducing an electronic audit and feedback system into current practice to improve hand hygiene compliance among front-line HCPs is subject to some uncertainty because it was based on indirect sources of evidence and a series of assumptions. The relationship between HCPs improving compliance with hand hygiene and reducing the background incidence of HCAIs is uncertain. Moreover, the model does not consider HCPs' adaptation to the electronic audit and feedback system and subsequent decline in compliance. Furthermore, the model does not distinguish between different types of pathogens causing HCAIs and the definition of HCAI would appear to vary between clinicians.

The model was populated with estimates for an 'average general hospital' and 'average patients' and does not consider the impact of other factors that may affect the results, such as different ward types, patients' age or co-morbidities, clinician specialties etc. By restricting the framework of the model to the average general hospital, the analysis does not consider the impact of improving hand hygiene compliance in specialist centres such as cancer hospitals, mental health and disability units and women's hospitals. However, there is no reason to assume that the findings from this study would be any different in a specialist hospital. Additionally, the analysis is limited to HCAIs among adult inpatients and does not consider paediatric patients, accident and emergency or outpatient departments. Neither does the model consider that patients may acquire a HCAI and be discharged from hospital before symptoms emerge. The model assumes that patients who do not die from a HCAI are discharged, 


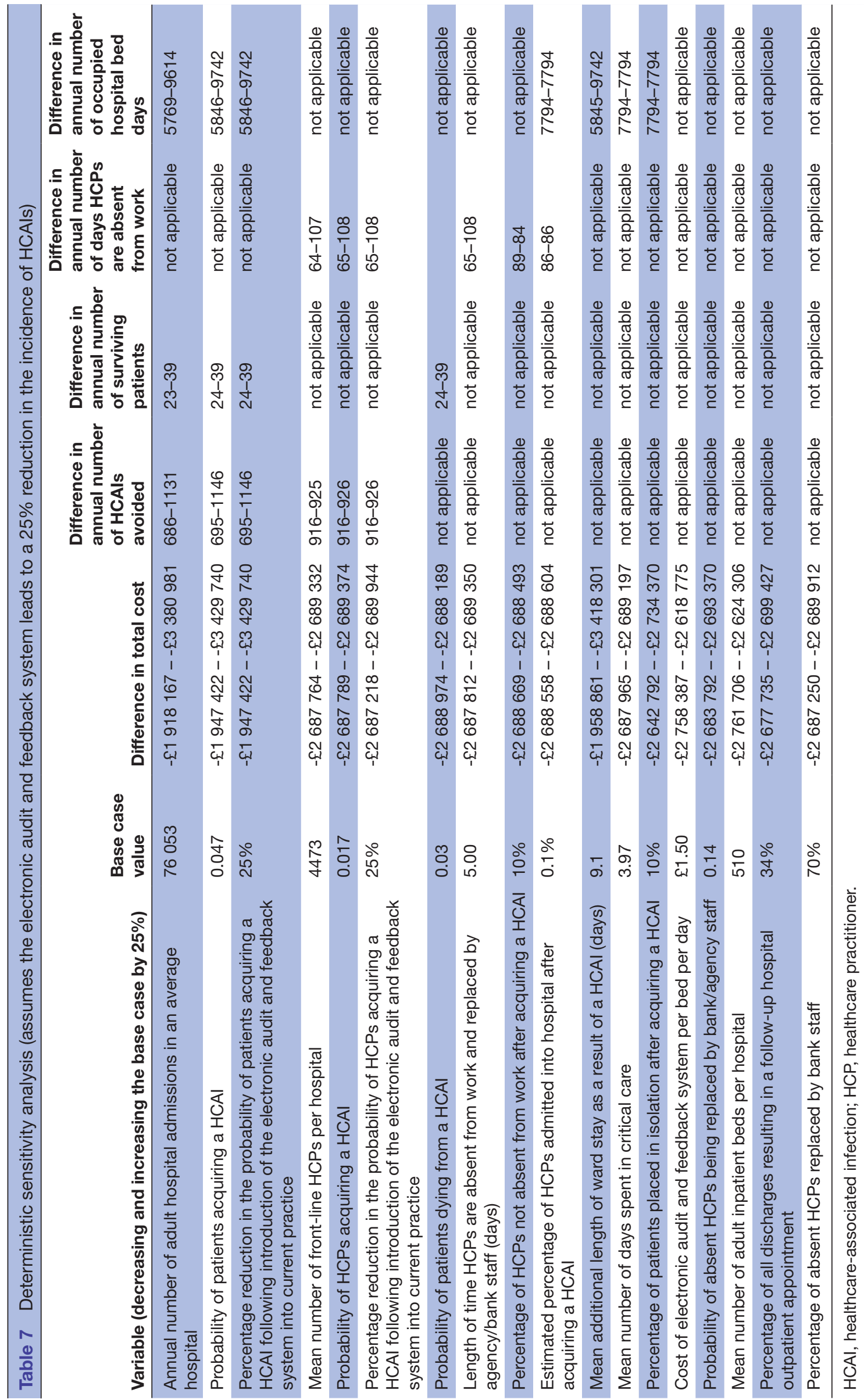


but in reality some patients may die from other reasons. The analysis excluded patients' costs and indirect costs incurred by society as a result of employed patients or HCPs taking time off work as a result of a HCAI. Additionally, estimating the value of improving hand hygiene overall is beyond the remit of this study. Notwithstanding this, the Authors chose to focus on electronic monitoring systems because of their higher degree of accuracy and current level of interest. The evidence shows that manual audits lead to a marked Hawthorne effect and other sources of bias (eg, postponing complicated clinical procedures until the auditor has gone).${ }^{16}$ The other method of measuring adherence is product consumption which is not very accurate and provides little information. ${ }^{17}$ Inaccuracies associated with manual hand hygiene audit are such that it is no longer considered the best approach and there is increasing interest in combining periodic manual audit with continuing electronic monitoring to give an overall picture of behaviour. ${ }^{5152}$ Clearly, a controlled study is required to compare the introduction of an electronic audit and feedback system into current practice to improve hand hygiene compliance with current practice in order to validate the outcomes of this analysis.

\section{Conclusion}

Within the study's limitations, the introduction of an electronic audit and feedback system into current practice to improve hand hygiene compliance among frontline HCPs in a hypothetical general hospital in England would afford the NHS a cost-effective intervention if it yielded a reduction in the incidence of HCAIs by $\geq 15 \%$.

Contributors JFG designed the study, managed the analyses, performed some analyses, checked all the other analyses and wrote the manuscript. TK conducted much of the analyses. DJG and NW scrutinised the analyses, suggested further analyses and helped interpret some of the findings. All the authors were involved in revising the manuscript and gave final approval. JFG is the guarantor of this work and, as such, had full access to all the data in the study and takes responsibility for the integrity of the data and the accuracy of the data analysis.

Funding This study was funded by Essity Hygiene and Health AB, Mölndal, Sweden. The study's sponsors had no involvement in the study design, the collection, analysis and interpretation of the data, the writing of this manuscript and the decision to submit this article for publication. The views expressed in this article are those of the authors, and not necessarily those of Essity Hygiene and Health.

Competing interests None declared.

Patient consent for publication Not required.

Provenance and peer review Not commissioned; externally peer reviewed.

Data availability statement All data relevant to the study are included in the article or uploaded as supplementary information.

Open access This is an open access article distributed in accordance with the Creative Commons Attribution Non Commercial (CC BY-NC 4.0) license, which permits others to distribute, remix, adapt, build upon this work non-commercially, and license their derivative works on different terms, provided the original work is properly cited, appropriate credit is given, any changes made indicated, and the use is non-commercial. See: http://creativecommons.org/licenses/by-nc/4.0/.

\section{ORCID iDs}

Julian F Guest http://orcid.org/0000-0003-0162-2007

Dinah Gould http://orcid.org/0000-0002-1506-9532

\section{REFERENCES}

1. World Health Organinization.A brief synopsis on patient safety, 2010. Available: http://www.euro.who.int/_data/assets/pdf_file/0015/ 111507/E93833.pdf?ua=1. 2018

2. National institute for Health and Care Excellence. HealthcareAssociated infections prevention and control, 2011. Available: https://www.nice.org.uk/guidance/ph36

3. Pratt RJ, Pellowe CM, Wilson JA, et al. epic2: national evidencebased guidelines for preventing healthcare-associated infections in NHS hospitals in England. J Hosp Infect 2007;65(Suppl 1):S1-59.

4. World Health Organization. WHO guidelines on hand hygiene in health care, 2009. Available: http://www.who.int/iris/handle/10665/ 44102 [Accessed 15 Jan 2019].

5. National Audit Office. Reducing healthcare associated in hospital in England, report by the comptroller and auditor General | HC 560 session 2008-2009, 2009. Available: https://www.nao.org.uk/wpcontent/uploads/2009/06/0809560.pdf. 2018

6. Health Protection Agency. English national point prevalence survey on healthcare-associated infections and antimicrobial use, preliminary data, 2011. Available: http://webarchive.nationalarchives. gov.uk/20140714085429tf_/http://www.hpa.org.uk/Publications/ InfectiousDiseases/AntimicrobialAndHealthcareAssociatedInfections/ 1205HCAIEnglishPPSforhcaiandamu2011 prelim/

7. World Health Organisation. Evidence of hand hygiene to reduce transmission and infections by multidrug resistant organisms in health-care settings. Available: http://www.who.int/gpsc/5may/ MDRO_literature-review.pdf

8. World Health Organization. Health care associated infections fact sheet. Available: http://www.who.int/gpsc/country_work/gpsc_ccisc_ fact sheet en.pdf

9. Gov.UK. Healthcare associated infections (HCAl): guidance, data and analysis, 2018. Available: https://www.gov.uk/government/ collections/healthcare-associated-infections-hcai-guidance-dataand-analysis [Accessed 24 Apr 2018]

10. Loveday HP, Wilson JA, Pratt RJ, et al. epic3: national evidencebased guidelines for preventing healthcare-associated infections in NHS hospitals in England. J Hosp Infect 2014;86(Suppl 1):S1-S70.

11. World Health Organization. My five moments for hand hygiene, 2009 Available: https://www.who.int/gpsc/5may/background/5moments/ en/ [Accessed 30 Jan 2019].

12. Ivers $\mathrm{NM}$, Sales $\mathrm{A}$, Colquhoun $\mathrm{H}$, et al. No more 'business as usual' with audit and feedback interventions: towards an agenda for a reinvigorated intervention. Implementation Sci 2014;9.

13. Gould DJ, Moralejo D, Drey N, et al. Interventions to improve hand hygiene compliance in patient care. Cochrane Database Syst Rev 2017;9.

14. Scherer AM, Reisinger HS, Goto M, et al. Testing a novel audit and feedback method for hand hygiene compliance: a multicenter quality improvement study. Infect Control Hosp Epidemiol 2019;40:89-94.

15. McCambridge J, Witton J, Elbourne DR. Systematic review of the Hawthorne effect: new concepts are needed to study research participation effects. J Clin Epidemiol 2014;67:267-77.

16. Srigley JA, Furness CD, Baker GR, et al. Quantification of the Hawthorne effect in hand hygiene compliance monitoring using an electronic monitoring system: a retrospective cohort study. BMJ Qual Saf 2014;23:974-80.

17. Gould DJ, Creedon S, Jeanes A, et al. Impact of observing hand hygiene in practice and research: a methodological reconsideration. $J$ Hosp Infect 2017;95:169-74.

18. Srigley JA, Gardam M, Fernie G, et al. Hand hygiene monitoring technology: a systematic review of efficacy. J Hosp Infect 2015;89:51-60.

19. Ward MA, Schweizer ML, Polgreen PM, et al. Automated and electronically assisted hand hygiene monitoring systems: a systematic review. Am J Infect Control 2014;42:472-8.

20. Pittet D, Hugonnet S, Harbarth $S$, et al. Effectiveness of a hospitalwide programme to improve compliance with hand hygiene. The Lancet 2000;356:1307-12.

21. NHS Digital. Hospital episode statistics on admissions stratified by hospital provider 2016-17. Available: https://digital.nhs.uk/data-andinformation/publications/statistical/hospital-admitted-patient-careactivity/2017-18

22. NHS England. Beds availability and occupancy data, 2018. Available: https://www.england.nhs.uk/statistics/statistical-work-areas/bedavailability-and-occupancy/

23. NHS Hospital \& Community Health Service (HCHS) monthly workforce statistics. Provisional statistics staff in trusts and CCGs, 2017. Available: https://digital.nhs.uk/data-and-information/ publications/statistical/nhs-workforce-statistics/nhs-workforcestatistics-july-2017-provisional-statistics 
24. European Centre for Disease Prevention and Control surveillance report. Point prevalence survey of healthcare associated infections and antimicrobial use in European acute care hospitals 20112012. Available: https://ecdc.europa.eu/sites/portal/files/media/ en/publications/Publications/healthcare-associated-infectionsantimicrobial-use-PPS.pdf

25. Cassini A, Plachouras D, Eckmanns T, et al. Burden of six healthcareassociated infections on European population health: estimating incidence-based Disability-Adjusted life years through a population Prevalence-Based modelling study. PLoS Med 2016;13:e1002150.

26. Health and Safety Executive. Health and safety statistics for the public sector services in Great Britain, 2017. Available: http://www. hse.gov.uk/statistics/industry/public-administration/public-services. pdf

27. Health \& Safety Executive website. Available: http://www.hse.gov.uk/ biosafety/healthcare.htm

28. Gould DJ, Drey NS, Chudleigh J, et al. Isolating infectious patients: organizational, clinical, and ethical issues. Am J Infect Control 2018;46:e65-9.

29. NHS Digital. Hospital episode statistics on admitted diagnosis 2016-17. Available: https://digital.nhs.uk/data-and-information/ publications/statistical/hospital-admitted-patient-care-activity/201617

30. NHS Digital. Hospital adult critical care activity 2016-17. Available: https://digital.nhs.uk/data-and-information/publications/statistical/ hospital-admitted-patient-care-activity/2016-17

31. NHS Improvement. Agency rules, 2018. Available: https:// improvement.nhs.uk/documents/2827/Agency_rules___2018_final_ draft.pdf

32. Wigglesworth $\mathrm{N}$, Gould D, Lindstrom $\mathrm{H}$, et al. Using an automated electronic hand hygiene device with performance feedback to increase hand hygiene adherence: an interrupted time series study. 11th annual conference of Infection Prevention Society, 2018.

33. King's Fund. Nhs Hospital bed numbers: past, present, future, 2017. Available: https://www.kingsfund.org.uk/publications/nhs-hospitalbed-numbers [Accessed 30 Jan 2019].

34. World Health Organization. Health worker occupational health. Available: https://www.who.int/occupational_health/topics/ hcworkers/en/ [Accessed 12 May 2019].

35. Fedeli U, Zanetti C, Saia B. Susceptibility of healthcare workers to measles, mumps rubella and varicella. $J$ Hosp Infect 2002;51:133-5.

36. Hyams PJ, Stuewe MC, Heitzer V. Herpes zoster causing varicella (chickenpox) in hospital employees: cost of a casual attitude. Am J Infect Control 1984;12:2-5.

37. Clark NM, Lynch JP. Influenza: epidemiology, clinical features, therapy, and prevention. Semin Respir Crit Care Med 2011;32:373-92.

38. Stewardson A, Sax H, Longet-Di Pietro S, et al. Impact of observation and analysis methodology when reporting hand hygiene data. J Hosp Infect 2011;77:358-9.

39. Boyce JM. Electronic monitoring in combination with direct observation as a means to significantly improve hand hygiene compliance. Am J Infect Control 2017;45:528-35.

40. K IK R, K M, E R, et al. The provision of alcohol-based products to improve compliance with hand hygiene. health technology assessment report 7. NHS quality improvement Scotland, 2005. Available: http://www.healthcareimprovementscotland.org/ previous_resources/hta_report/hta_7.aspx [Accessed 16 Jan 2019].

41. Luangasanatip N, Hongsuwan M, Lubell $\mathrm{Y}$, et al. Cost-Effectiveness of interventions to improve hand hygiene in healthcare workers in middle-income Hospital settings: a model-based analysis. $J$ Hosp Infect 2018;100:165-75

42. Chen $\mathrm{Y}-\mathrm{C}$, Sheng $\mathrm{W}-\mathrm{H}$, Wang J-T, et al. Effectiveness and limitations of hand hygiene promotion on decreasing healthcare-associated infections. PLoS One 2011;6:e27163.

43. Huis A, Hulscher M, Adang E, et al. Cost-Effectiveness of a team and leaders-directed strategy to improve nurses' adherence to hand hygiene guidelines: a cluster randomised trial. Int J Nurs Stud 2013;50:518-26.

44. Weber DJ, Rutala WA, Miller MB, et al. Role of hospital surfaces in the transmission of emerging health care-associated pathogens: norovirus, Clostridium difficile, and Acinetobacter species. Am J Infect Control 2010;38(5 Suppl 1):S25-S33.

45. Derde LPG, Cooper BS, Goossens H, et al. Interventions to reduce colonisation and transmission of antimicrobial-resistant bacteria in intensive care units: an interrupted time series study and cluster randomised trial. Lancet Infect Dis 2014;14:31-9.

46. Wille I, Mayr A, Kreidl P, et al. Cross-Sectional point prevalence survey to study the environmental contamination of nosocomial pathogens in intensive care units under real-life conditions. $J$ Hosp Infect 2018:98:90-5.

47. Rosenthal VD, Guzman S, Safdar N. Effect of education and performance feedback on rates of catheter-associated urinary tract infection in intensive care units in Argentina. Infect Control Hosp Epidemiol 2004;25:47-50.

48. Gastmeier P, Geffers C. Prevention of ventilator-associated pneumonia: analysis of studies published since 2004. J Hosp Infect 2007:67:1-8.

49. Shah N, Castro-Sánchez E, Charani E, et al. Towards changing healthcare workers' behaviour: a qualitative study exploring noncompliance through appraisals of infection prevention and control practices. J Hosp Infect 2015;90:126-34.

50. Ivers N, Jamtvedt G, Flottorp S, et al. Audit and feedback: effects on professional practice and healthcare outcomes. Cochrane Database Syst Rev 2012;154.

51. Marra AR, Edmond MB. Hand hygiene: state-of-the-art review with emphasis on new technologies and mechanisms of surveillance. Curr Infect Dis Rep 2012;14:585-91.

52. Masroor N, Doll M, Stevens M, et al. Approaches to hand hygiene monitoring: from low to high technology approaches. Int $\mathrm{J}$ Infect Dis 2017;65:101-4.

53. National Institute of Economic and Social Research. Use of agency staff in the public sector, 2017. Available: https://www.niesr.ac.uk/ sites/default/files/publications/NIESR_agency_working_report_final. pdf

54. NHS Digital. Hospital outpatient activity, 2016-17. provider level analysis. Available: https://digital.nhs.uk/data-and-information/ publications/statistical/hospital-outpatient-activity/hospitaloutpatient-activity-2016-17

55. NHS Digital. Hospital episode statistics on first outpatient attendances 2016-17. Available: https://digital.nhs.uk/data-andinformation/publications/statistical/hospital-outpatient-activity/ hospital-outpatient-activity-2016-17

56. NHS Digital. Hospital episode statistics on outpatient attendances 2016-17. main specialty by attendance type. Available: https:// digital.nhs.uk/data-and-information/publications/statistical/hospitaloutpatient-activity/hospital-outpatient-activity-2016-17

57. NHS Digital. National schedule of reference costs 2016-17. Available: https://improvement.nhs.uk/resources/reference-costs/

58. PSSRU. Unit costs of health and social care, 2017. Available: https:// www.pssru.ac.uk/project-pages/unit-costs/unit-costs-2017/ 